\title{
Lissencephaly with cerebellar hypoplasia type B
}

INSERM

\section{Source}

INSERM. (1999). Orphanet: an online rare disease and orphan drug data base.

Lissencephaly with cerebellar hypoplasia type B. ORPHA:100012

Lissencephaly with cerebellar hypoplasia type B (LCHb) is a form of lissencephaly with cerebellar hypoplasia characterized by subtle microcephaly, hypotonia and neurological and cognitive development delay. Hippocampal malformation is a characteristic imaging feature of LCHb. 